\title{
Rapidly progressive cardiac failure due to lymphomatous infiltration of the myocardium
}

\author{
D.R. Gouldesbrough and P.J. Carder \\ Department of Pathology, University of Edinburgh Medical School, Teviot Place, Edinburgh EH8 9AG, UK.
}

\begin{abstract}
Summary: A case of myocardial infiltration by lymphoma causing rapidly progressive cardiac failure is described. The clinical and pathological features are detailed and the rarity of this mode of presentation of cardiac involvement by lymphoma is emphasized.
\end{abstract}

\section{Introduction}

Cardiac involvement occurs in $20-25 \%$ of patients with malignant lymphoma. ${ }^{1,2}$ This is usually in the context of widespread disease although rare primary lymphomas of the myocardium have been reported. ${ }^{3,4}$ The pattern of infiltration of the heart varies but the presence of lymphoma is usually asymptomatic in life and only diagnosed at autopsy. ${ }^{2}$ We report a case of rapidly progressive cardiac failure due to lymphomatous infiltration of the left ventricular myocardium.

\section{Case report}

A 49 year old male was admitted as an emergency with a one week history of progressive dyspnoea, initially on exertion and then at rest. Associated with this were a cough productive of frothy sputum, abdominal distension and ankle swelling.

His past medical history was of follicular lymphoma of the spleen and adjacent lymph nodes diagnosed in 1981 with transformation to a high grade diffuse centroblastic lymphoma in 1987. The latter was demonstrated in bone marrow, testis and cerebrospinal fluid and he was treated with several pulses of chemotherapy. He was a non-insulin dependent diabetic.

On examination he was dyspnoeic with peripheral and pulmonary oedema, hepatomegaly and ascites noted. Investigations revealed anaemia and thrombocytopenia which were treated with red cell and platelet transfusions.

Despite the clinically severe cardiac failure with a very poorly functioning left ventricle, no specific

Correspondence: D.R. Gouldesbrough, B.Sc., M.B., Ch.B. Accepted: 20 February 1989 electrocardiographic changes were noted. His condition was complicated by gastrointestinal bleeding. Inotropic support was given for the left ventricular malfunction and transfusions of whole blood and platelets were continued. The patient did not respond to these measures and he died in severe cardiac failure two days after admission.

\section{Pathology}

The splenectomy in 1981 yielded an enlarged spleen substantially replaced by a follicular lymphoma. Bone marrow biopsy and unilateral orchidectomy during 1987 showed transformation to a high grade diffuse centroblastic lymphoma. The latter is illustrated in Figure 1. A cerebrospinal fluid sample taken after an episode of ataxia contained abnormal lymphoid cells showing kappa light chain restriction consistent with central nervous system involvement with lymphoma.

At autopsy the significant findings were in the heart, lungs, bone marrow and small bowel. The heart weighed $368 \mathrm{~g}$. Both atria were normal and the right ventricle was mildly dilated. Approximately $40 \%$ of the circumference of the left ventricular myocardium was replaced by firm, pale tissue. This extended from the pericardium where it was most pronounced, into the myocardium and focally to the endocardial surface. Histology showed infiltration of the myocardium and pericardium by a large celled non-Hodgkin's lymphoma of centroblastic type (Figure 2). The coronary arteries were only minimally atheromatous and no ischaemic damage to the myocardium was detected. No myocardial or endocardial changes indicating long standing heart failure were found and there was no outflow tract obstruction.

Both lungs were congested and severely C) The Fellowship of Postgraduate Medicine, 1989 
a

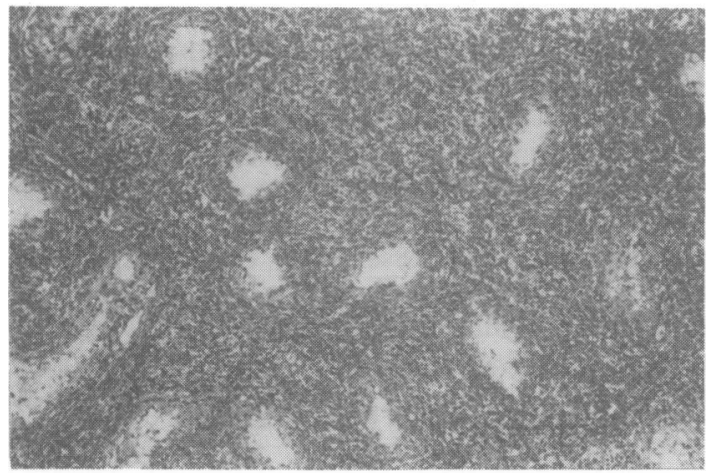

b

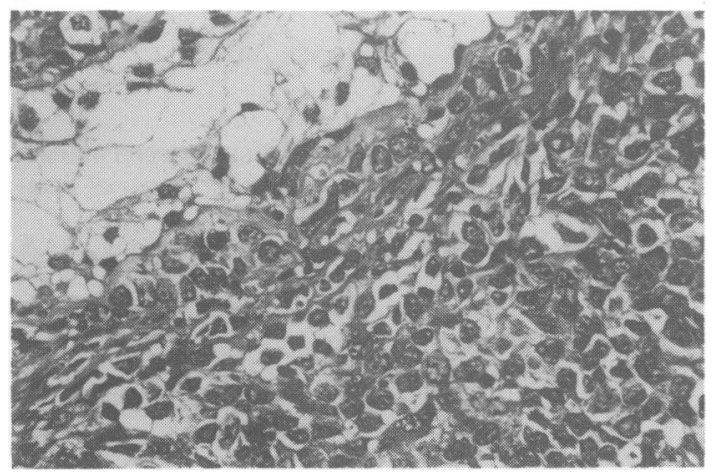

Figure 1 (a) Low power view of infiltration of the testis by lymphoma. Residual seminiferous tubules are seen $(\mathbf{H}$ $\& \mathrm{E} \times 25$ ). (b) A high power view to illustrate the high grade, large celled nature of the lymphoma (H \& $\mathrm{E} \times 250$ ).

oedematous. Lymphomatous infiltration was present bilaterally in a characteristic interstitial distribution with peribronchial and perivascular accentuation. No evidence of pulmonary hypertension was found.

Extensive bone marrow infiltration was demonstrated and many discreet ulcerated nodules of lymphoma were present within the wall of the small bowel. Bleeding had occurred from the latter and from several acute gastric and duodenal erosions.

\section{Discussion}

Cardiac involvement in systemic lymphoma is a relatively common occurrence accounting for $9 \%$ of cases of metastatic malignancy to the heart in autopsy studies. ${ }^{2}$ The reported frequency of infiltration of the heart in patients with systemic lymphoma varies from $9 \%$ to $24 \%$. $^{1,2}$ Despite this, symptoms and signs a

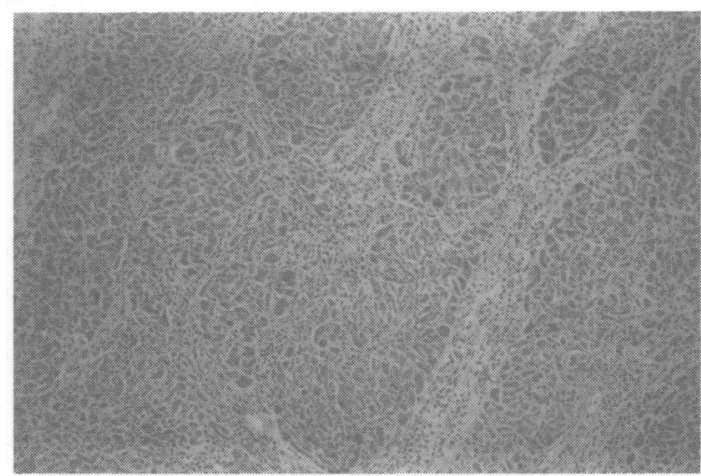

b

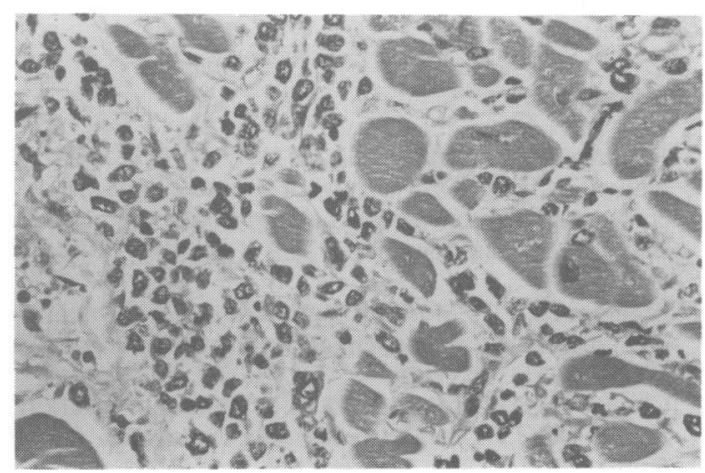

Figure 2 (a) Infiltration of the myocardium by lymphoma (H \& E $\times 25$ ). (b) A high power view to demonstrate the pleomorphism and large size of the infiltrating cells (H \& E × 250).

reflecting cardiac involvement are distinctly unusual. McDonnell and co-workers ${ }^{2}$ found only 2 patients in their series of 150 cases of lymphoma who died a cardiac death, with the diagnosis of lymphomatous infiltration of the heart apparent prior to death in only one. Six of the 196 patients reported by Roberts and co-workers ${ }^{1}$ had evidence of cardiac dysfunction but the underlying cause was inapparent until autopsy examination.

The pattern of cardiac involvement varies from nodular intracavitary growths causing outflow tract obstruction $^{5,6}$ to a more infiltrative spread in usually the right but also the left side of the heart. ${ }^{4} \mathrm{~A}$ predominantly pericardial distribution has also been reported. To explain these different patterns of infiltration three modes of spread to the heart have been suggested: retrograde lymphatic, haematogenous and direct extension. ${ }^{2}$ The last of these has been found most destructive with the highest frequency of cardiac dysfunction. The majority of lym- 
phomas involving the heart are non-Hodgkin's in type.

The case we describe is unusual in three respects. Firstly the rapidity of progression of the cardiac failure is rare. Similar intractable cardiac failure has been reported with primary and metastatic lymphoma ${ }^{3,4,7,8}$ in the heart but it is distinctly uncommon. Secondly, the pattern of infiltration of the heart suggests a retrograde lymphatic mode of spread. ${ }^{2}$ McDonnell et al. ${ }^{2}$ found this pattern did not produce clinical signs but rather direct extension from mediastinal disease was most destructive. We found no evidence of such spread in our case. Thirdly, the majority of reported cases giving rise to symptoms involved the right side of the heart rather than the left as in our patient.

\section{References}

1. Roberts, W.C., Glancy, D.L. \& De Vita, V.T. Heart in malignant lymphoma (Hodgkin's disease, lymphosarcoma, reticulum cell sarcoma and mycosis fungoides). A study of 196 autopsy cases. Am J Cardiol 1968, 22: 85-107.

2. McDonnell, P.J., Mann, R.B. \& Bulkley, B.H. Involvement of the heart by malignant lymphoma. Cancer 1982, 49: 944-951.

3. Chou, S.T., Arkles, L.B., Gill, G.D., Pinkus, N., Parkin, A. \& Hicks, J.D. Primary lymphoma of the heart. A case report. Cancer 1983, 52: 744-747.

4. Constantino, A., West, T.E., Gupta, M. \& Loghmanee, P. Primary cardiac lymphoma in a patient with acquired immune deficiency syndrome. Cancer 1987, 60: $2801-2805$.

5. Kuo, T.T., Yang, C.P., Lin, C.H. \& Chang, C.H. Lymphoblastic lymphoma presenting as a huge intracavitary cardiac tumour causing heart failure. Pediatr Pathol 1987, 7: 341-349.
We describe a very uncommon cause of rapidly progressive, intractable cardiac failure. Chemotherapy for lymphomatous infiltration of the heart is at least potentially curative although cardiotoxicity may be a problem with, especially, doxorubicin. ${ }^{9}$ Scott and Garvin, ${ }^{10}$ writing in 1938 , pointed out that the most important sign of cardiac invasion by malignant tumour is congestive cardiac failure without other apparent cause. This case serves to emphasize this point and illustrates a need for a high index of suspicion of cardiac infiltration in patients with a known lymphoma who develop rapid congestive heart failure.

6. Johnston, I.D. \& Popple, A.W. Right ventricular outflow tract obstruction secondary to small intestinal lymphoma. Br Heart J 1980, 43: 593-596.

7. Miyazaki, T., Yoshida, T., Mori, H., Handa, S. \& Nakamura, Y. Intractable heart failure, conduction disturbance and myocardial infarction by massive myocardial invasion of malignant lymphoma. J Am Coll Cardiol 1985, 6: 937-941.

8. Molajo, A.O., McWilliam, L., Ward, C. \& Rahman, A Cardiac lymphoma: an unusual case of myocardial perforation - clinical, echocardiographic, haemodynamic and pathological features. Eur Heart J 1987, 8: $549-552$.

9. Billingham, M.E. Some recent advances in cardiac pathology. Hum Pathol 1979, 10: 367-386.

10. Scott, R.W. \& Garvin, C.F. Tumours of the heart and pericardium. Am Heart $J$ 1938, 17: 431-436. 